\title{
ESTIMATES FOR NONANALYTIC CUSP FORMS
}

\author{
DAVID JOHNSON
}

\begin{abstract}
Estimates for the magnitude of nonanalytic cusp forms are obtained via the theory of the Selberg transformation and Fourier coefficient estimates.
\end{abstract}

1. Introduction. Let $\Gamma$ be a finitely generated Fuchsian group of the first kind, acting on $\nvdash=\{\tau / \tau=x+i y, y>0\}$. Let $\mathcal{F}$ denote a fundamental domain of $\Gamma$ with inequivalent parabolic cusps $s_{1}, \ldots, s_{\rho}$. We consider the nonanalytic normalized eigenfunctions for $\Gamma$, solutions of the eigenvalue problem

$$
y^{2}\left(u_{x x}+u_{y y}\right)+\lambda u=0
$$

with $u \in L_{2}(\Gamma \mid \not{H})$, the $\Gamma$-invariant functions on $\mathscr{H}$ with finite norm given by the scalar product

$$
\langle f, g\rangle \doteq \int_{\mathcal{F}} f(\tau) \overline{g(\tau)}(d \tau), \quad(d \tau)=y^{-2} d x d y .
$$

We assume further that our eigenfunctions are cusp forms. This requires that $\lambda=\lambda_{j}=t_{j}\left(1-t_{j}\right), t_{j}=1 / 2+i r_{j}, j=1,2,3, \ldots$, belong to the discrete spectrum of the non-Euclidean Laplace operator in (1.1). These eigenfunctions, $e_{j}(\tau)$, may be characterized as follows. Let $A_{i}, i=1, \ldots, \rho$, be chosen in $\mathrm{SL}_{2}(\mathbf{R})$ so that $A_{i} s_{i}=\infty, A_{i} \Gamma_{i} A_{i}^{-1}=\left\{\left(\begin{array}{ll}1 & n \\ 0 & 0\end{array}\right) \mid n \in \mathbf{Z}\right\}$, where $\Gamma_{i} \subset \Gamma$ is the stabilizer group of $s_{i}$. If $\tau_{k}=x_{k}+i y_{k}=A_{k} \tau$ for $k=1, \ldots, \rho$, then each cusp form has expansions of the form

$$
e_{j}(\tau)=\sum_{n \neq 0} \beta_{n, j}^{k} y_{k}^{1 / 2} K_{i r_{j}}\left(2 \pi|n| y_{k}\right) e^{2 \pi i n x_{k}},
$$

where $K_{i r}$ is the Bessel function. The cusp forms are characterized among the $L_{2}(\Gamma \mid \not{H})$ eigenfunctions by the vanishing of the constant (in $x_{k}$ ) term in the Fourier expansion at all cusps. Aside from some special examples for congruence subgroups of the modular group [3, p. 146], no cusp forms are explicitly known.

In what follows, $A \ll B, A=O(B)$ will mean $|A| \leq$ const $|B|$ and $C(.$.$) will$ indicate various constants depending on the indicated parameters. It is clear from the expansions (1.2) that $e_{j}(\tau) \rightarrow 0$ as $\tau \rightarrow s_{i}$ in $F$. It follows that $e_{j}(\tau)$ is bounded on $\mathcal{F}$. A bound of the form

$$
\left|e_{j}(\tau)\right| \ll\left(1+r_{j}^{2}\right),
$$

which is uniform in $\tau$, follows from the theory of the resolvent kernel for the Laplace operator (see [5, p. 274]). This paper contains improvements on the results given above.

Received by the editors September 29, 1983.

1980 Mathematics Subject Classification. Primary $10 \mathrm{D} 05$. 
Select $C>0$ large enough so that for any $\tau$ in $₹$ there is at most one $i$ so that $y_{i} \geq C$. Define $\kappa=\kappa(\tau)$ as follows:

$$
\kappa= \begin{cases}i & \text { if } y_{i} \geq C, \\ 1 & \text { if } y_{i}<C \text { for } 1 \leq i \leq \rho .\end{cases}
$$

It is clear that there is $C_{0}>0$ so that $y_{\kappa} \geq C_{0}$ for any $\tau$ in $\mathcal{F}$.

THEOREM 1. If $1 / 2<\alpha \leq 3 / 4$ and $\kappa=\kappa(\tau)$, then

$$
\left|e_{j}(\tau)\right|<C(\alpha)\left(1+r_{j}^{2 \alpha}\right) y_{\kappa}^{3 / 2-2 \alpha} .
$$

In particular, the bound $e_{j}(\tau) \ll\left(1+r_{j}^{3 / 2}\right)$ holds uniformly in $\tau$, while for $\tau$ fixed

$$
\left|e_{j}(\tau)\right|<C(\tau, \varepsilon)\left(1+r_{j}^{1+\varepsilon}\right)
$$

holds for any given $\varepsilon>0$.

The following inequality is better if $\tau$ approaches a cusp.

THEOREM 2. If $y_{\kappa}>r_{j} / 8$ and $\kappa=\kappa(\tau)$, then

$$
e_{j}(\tau) \ll e^{\pi r_{j} / 2} r_{j}^{1 / 2} e^{-2 \pi y_{\kappa}} y_{\kappa}^{-1 / 2} .
$$

Theorem 2 is the result of a simple estimate for the coefficients in the expansions (1.2). The estimate, which may be of some independent interest, is given as Theorem 3 in the next section. The first theorem results from using Selberg's theory of point pair invariants to construct a kernel with a stronger singularity and large eigenvalues than the resolvent kernel.

2. Poincaré series. In this section we state results about Poincaré series and Eisenstein series that will be needed later.

For $M \in \Gamma$ define

$$
\tau_{l, M}=x_{l, M}+i y_{l, M} \doteq A_{l} M A_{l}^{-1} \tau_{l}=A_{l} M \tau
$$

for $l=1, \ldots, \rho$. The Poincaré series for the $l$ th cusp are defined by

$$
U_{n}^{l}(\tau ; s) \doteq \sum_{\Gamma_{\iota} \backslash \Gamma} e^{-2 \pi|n| y_{l, M}+2 \pi i n x_{l, M}} y_{l, M}^{s}
$$

for $\sigma>1, s=\sigma+i t$. The sum is over representatives of left $\Gamma_{l}$ cosets of $\Gamma$. The Eisenstein series for the $l$ th cusp is defined to be $U_{0}^{l}(\tau ; s)$. The Eisenstein series has an expansion at the $k$ th cusp of the form (see [4, p. 24])

$$
\begin{aligned}
U_{0}^{l}(\tau ; s)= & \delta_{k, l} y_{k}^{s}+\phi_{k}^{l}(s) y_{k}^{1-s} \\
& +2 \pi^{s} \Gamma(s)^{-1} \sum_{m \neq 0}|m|^{s-1 / 2} L_{k, m}^{l}(s) K_{s-1 / 2}\left(2 \pi|m| y_{k}\right) e^{2 \pi i m x_{k}}
\end{aligned}
$$

where

$$
\delta_{k, l}= \begin{cases}1, & k=l, \\ 0, & k \neq l,\end{cases}
$$

$K_{s-1 / 2}$ is the Bessel function and $\Gamma(s)$ is the gamma function. The coefficients are given by

$$
L_{k, m}^{l}(s)=\sum_{(c, d)} e^{2 \pi i m d / c} c^{-2 s}, \quad \sigma>1
$$




$$
\phi_{k}^{l}(s)=\sqrt{\pi} \Gamma\left(s-\frac{1}{2}\right) \Gamma(s)^{-1} L_{k, 0}^{l}(s),
$$

where $\left(\begin{array}{ll}* & * \\ c & d\end{array}\right)=A_{l}^{-1} M A_{k}^{-1}$, with $M$ from $\Gamma_{l} \backslash \Gamma$. The Bessel function satisfies

$$
K_{s-1 / 2}(x) \ll x^{-1 / 2} e^{-x}
$$

uniformly for $1 \leq s \leq 2$ or for $x \gg t$, where $s=1 / 2+i t$. It is known from the theory of Eisenstein series that $L_{k, 0}^{l}(s)$ has at worst a simple pole at $s=1$. Thus for $1<s \leq 2$

$$
\left|L_{k, m}^{l}(s)\right| \leq L_{k, 0}^{l}(s) \ll(s-1)^{-1} .
$$

It follows from this, (2.2) and (2.3) that

$$
\begin{aligned}
U_{0}^{l}(\tau ; s)- & \delta_{k, l} y_{k}^{s} \ll \delta_{k, l} y_{\kappa}^{s}-\delta_{k, l} y_{k}^{s}+(s-1)^{-1} y_{\kappa}^{1-s} \\
& +(s-1)^{-1} \sum_{m \neq 0}|m|^{s-1 / 2}\left|K_{s-1 / 2}\left(2 \pi|m| y_{\kappa}\right)\right| \\
\ll & (s-1)^{-1} \quad(\kappa=\kappa(\tau))
\end{aligned}
$$

uniformly for $1<s \leq 2$, and $\tau \in \mathcal{F}$.

We can now establish the estimate for the Fourier coefficients of the cusp forms.

THEOREM 3. $\beta_{m, j}^{l} \ll e^{\pi r_{j} / 2}\left(r_{j}+1\right)^{1 / 2}|m|^{1 / 2} \log (|m|+5)$.

ProOF. The Petersson scalar product formula gives

$$
\begin{aligned}
\left\langle U_{m}^{l}(\tau ; s), e_{j}(\tau)\right\rangle= & \bar{\beta}_{m, j}^{l} \pi^{1 / 2}(\pi|m|)^{1 / 2-s} 2^{1-2 s} \\
& \times \Gamma\left(s-i r_{j}-1 / 2\right) \Gamma\left(s+i r_{j}-1 / 2\right) \Gamma(s)^{-1}
\end{aligned}
$$

(see [4, p. 57]); note that in [4] the Poincaré series differ from the $U_{n}^{l}$ by a factor depending on $s)$. Pick $s=1+1 / \log (|m|+5)+i r_{j}$, apply Stirling's estimate and the Schwarz inequality. It follows that

$$
\beta_{m, j}^{l} \ll|m|^{1 / 2} e^{\pi r_{j} / 2}\left(r_{j}+1\right)^{1 / 2}\left\langle U_{m}^{l}, U_{m}^{l}\right\rangle^{1 / 2} .
$$

Since by (2.4)

$$
\begin{aligned}
\left|U_{m}^{l}(\tau, s)\right| & \leq e^{-2 \pi y_{l}} y_{l}^{\sigma}+\sum_{M \neq \mathrm{id}} y_{l, M}^{\sigma} \\
& =e^{-2 \pi y_{l}} y_{l}^{\sigma}+\left(U_{0}^{l}\left(\tau^{\prime} ; \sigma\right)-y_{l}^{\sigma}\right) \ll(\sigma-1)^{-1},
\end{aligned}
$$

we have $\left\langle U_{m}^{l}, U_{m}^{l}\right\rangle \ll \log ^{2}(|m|+5)$ with the choice of $s$ given above.

Theorem 2 is an immediate consequence of Theorem $3,(2.3)$ and (1.2).

3. Estimation of the point pair invariant. Selberg's theory shows us that the $e_{j}$ satisfy integral equations of the form

$$
h\left(r_{j}\right) e_{j}(\tau)=\int_{\mathscr{K}} k\left(\tau, \tau^{\prime}\right) e_{j}\left(\tau^{\prime}\right)\left(d \tau^{\prime}\right)
$$

provided that $k$ is a point pair invariant. This means that $k$ is a function of the hyperbolic distance $d\left(\tau, \tau^{\prime}\right)$, satisfying suitable conditions of growth and regularity. If (see [6, p. 72])

$$
t\left(\tau, \tau^{\prime}\right) \doteq\left|\tau-\tau^{\prime}\right|^{2} / y y^{\prime}=2 \cosh d\left(\tau, \tau^{\prime}\right)-2
$$


then $h$ and $k$ are related by

$$
\begin{aligned}
& g(u) \doteq \frac{1}{2 \pi} \int_{-\infty}^{+\infty} e^{-i r u} h(r) d r \\
& g(u) \doteq Q(w), \quad w=2 \cosh u-2 \\
& k(t) \doteq-\frac{1}{\pi} \int_{t}^{\infty}(w-t)^{-1 / 2} d Q(w), \\
& k\left(\tau, \tau^{\prime}\right)=k\left(t\left(\tau, \tau^{\prime}\right)\right) .
\end{aligned}
$$

We will construct $k$ by choosing $h(r)=\left(4+r^{2}\right)^{-p-1 / 2}$ with $0<p<1 / 2$. This gives, by (3.1),

$$
g(u)=4^{-p} \pi^{-1 / 2} \Gamma(p+1 / 2)^{-1} u^{p} K_{p}(2 u),
$$

where $\Gamma$ is the gamma function and $K_{p}$ is the Bessel function. The series expansion $K_{p}$ in powers of $u$ gives

$$
u^{p} K_{p}(2 u)=\pi \csc \pi p\left\{\Gamma(1-p)^{-1}-\Gamma(p+1) u^{2 p}+\cdots\right\} .
$$

Termwise differentiation shows

$$
g^{\prime}(u) \ll u^{2 p-1}, \quad g^{\prime \prime}(u) \ll u^{2 p-2}
$$

for $u \rightarrow 0$. The asymptotic estimate for $K_{p}$ implies

$$
g^{\prime}(u) \ll u^{p-1 / 2} e^{-2 u}, \quad g^{\prime \prime}(u) \ll u^{p-1 / 2} e^{-2 u}
$$

for $u \rightarrow \infty$.

The relation between $u$ and $w$ in (3.1) gives

$$
u= \begin{cases}w^{1 / 2}+O(w), & w \rightarrow 0 \\ \log \left(1+w+O\left(w^{-1}\right)\right), & w \rightarrow \infty\end{cases}
$$

It follows that

$$
\begin{aligned}
& Q^{\prime}(w) \ll \begin{cases}w^{p-1}, & 0<w<1, \\
(1+w)^{-11 / 4}, & w \geq 1,\end{cases} \\
& Q^{\prime \prime}(w) \ll \begin{cases}w^{p-2}, & 0<w<1, \\
(1+w)^{-15 / 4}, & w \geq 1 .\end{cases}
\end{aligned}
$$

It is now possible to estimate the function $k$, where by $(3.1)$

$$
k(t)=-\frac{1}{\pi} \int_{0}^{\infty} Q^{\prime}(w+t) w^{-1 / 2} d w .
$$

If $0<t<1$, then by $(3.6)$

$$
\begin{aligned}
k(t) & \ll t^{p-1 / 2} \int_{0}^{t^{-1}}(w+1)^{p-1} w^{-1 / 2} d w+\int_{1}^{\infty}(1+w+t)^{-2} w^{-1 / 2} d w \\
& \ll t^{p-1 / 2}+t^{p-1 / 2} \int_{1}^{t^{-1}} w^{p-3 / 2} d w \ll t^{p-1 / 2}
\end{aligned}
$$


If $t \geq 1$ then

$$
k(t) \ll \int_{0}^{\infty}(1+w+t)^{-11 / 4} w^{-1 / 2} d w \ll t^{-2} .
$$

A similar estimate for $k^{\prime}$ can be obtained in the same way from (3.6). In summary, if

$$
\begin{aligned}
& k_{1}(t) \doteq \begin{cases}t^{p-1 / 2}, & 0<t<1, \\
t^{-2}, & t \geq 1,\end{cases} \\
& k_{1}^{\prime}(t) \doteq \begin{cases}t^{p-3 / 2}, & 0<t<1, \\
t^{-3}, & t \geq 1,\end{cases}
\end{aligned}
$$

then the following lemma holds.

LEMMA $1 . k(t) \ll k_{1}(t), k^{\prime}(t) \ll k_{1}^{\prime}(t)$.

4. Lemmas for Theorem 1. Define

$$
\begin{aligned}
& K\left(\tau, \tau^{\prime}\right) \doteq \sum_{\gamma \in \Gamma} k\left(\tau, \gamma \tau^{\prime}\right)=\sum_{\Gamma_{i} \backslash \Gamma} \sum_{n=-\infty}^{+\infty} k\left(\tau_{i}, U^{n} \tau_{i, M}\right) \\
& k_{0}\left(\tau, \tau^{\prime}\right) \doteq \int_{-\infty}^{+\infty} k\left(\tau, U^{x} \tau^{\prime}\right) d x, \\
& K_{i}\left(\tau, \tau^{\prime}\right) \doteq \sum_{\Gamma_{i} \backslash \Gamma} k_{0}\left(\tau_{i}, \tau_{i, M}\right), \quad \tau=x+i y, \tau^{\prime}=x^{\prime}+i y^{\prime}
\end{aligned}
$$

and $i=1, \ldots, \rho$. The sums indicated by $\Gamma_{i} \backslash \Gamma$ are over all cosets, $\Gamma_{i} M$, and $U^{x} \doteq\left(\begin{array}{ll}1 & x \\ 0 & 1\end{array}\right)$. We seek to estimate the kernel $K$, with the contribution from $K_{\kappa}$ removed. The following lemmas furnish preparation for this estimate.

LEMMA 2. $k_{0}\left(\tau, \tau^{\prime}\right) \ll y^{-1 / 8} y^{\prime 9 / 8}$.

PROOF. If $7 y / 8 \leq y^{\prime} \leq 9 y / 8$, then Lemma 1 implies

$$
k_{0}\left(\tau, \tau^{\prime}\right) \ll\left(y y^{\prime}\right)^{1 / 2} \int_{-\infty}^{+\infty} k_{1}\left(t^{2}\right) d t \ll\left(y y^{\prime}\right)^{1 / 2} \ll y^{-1 / 8} y^{\prime 9 / 8} .
$$

Now assume $y^{\prime}<7 y / 8$ or $9 y / 8<y^{\prime}$. It follows that $\left(y-y^{\prime}\right)^{2} / y y^{\prime}>1 / 72$, and now by Lemma 1 ,

$$
k\left(\tau, \tau^{\prime}\right) \ll\left(\left|\tau-\tau^{\prime}\right| / y y^{\prime}\right)^{-2}
$$

This gives

$$
k_{0}\left(\tau, \tau^{\prime}\right) \ll\left(y y^{\prime}\right)^{2} \int_{-\infty}^{+\infty}\left\{\left(x-x^{\prime}-t\right)^{2}+\left(y-y^{\prime}\right)^{2}\right\}^{-2} d t \ll\left(y y^{\prime}\right)^{2}\left|y-y^{\prime}\right|^{-3}
$$

If $y^{\prime}>9 y / 8$ then

$$
\left(y^{\prime} y\right)^{2}\left|y-y^{\prime}\right|^{-3}=y^{2} y^{\prime-1}\left|1-y / y^{\prime}\right|^{-3} \ll y^{2} y^{\prime-1} \ll y^{-1 / 8} y^{\prime 9 / 8} .
$$

If $y^{\prime}<7 y / 8$ then similarly

$$
y^{-1} y^{2}\left|1-y^{\prime} / y\right|^{-3} \ll y^{-1} y^{2} \ll y^{-1 / 8} y^{\prime 9 / 8} .
$$

This completes the proof of Lemma 2. 
LEMMA 3. If $7 y / 8 \leq y^{\prime} \leq 9 y / 8$ and $y \neq y^{\prime}$ then

$$
\sum_{n=-\infty}^{+\infty} k\left(\tau, U^{n} \tau\right) \ll y^{-1 / 8} y^{9 / 8}+\left(y y^{\prime}\right)^{1 / 2-p} \sum_{|n| \leq 3}\left\{\left(x-x^{\prime}-n\right)^{2}+\left(y-y^{\prime}\right)^{2}\right\}^{p-1 / 2} .
$$

If $y^{\prime}<7 y / 8$ or $y^{\prime}>9 y / 8$ then

$$
\sum_{n=-\infty}^{+\infty} k\left(\tau, U^{n} \tau^{\prime}\right) \ll y^{-1 / 8} y^{9 / 8} .
$$

ProOF. Let $7 y / 8 \leq y^{\prime} \leq 9 y / 8$ and $y \neq y^{\prime}$. Lemma 1 implies

$$
\begin{aligned}
\sum_{n} k\left(\tau, U^{n} \tau^{\prime}\right) \ll & \left(y y^{\prime}\right)^{1 / 2-p} \sum_{|m| \leq 3}\left\{\left(x-x^{\prime}-m\right)^{2}+\left(y-y^{\prime}\right)^{2}\right\}^{p-1 / 2} \\
& +\left(y y^{\prime}\right)^{1 / 2-p} \sum_{1 \leq\left|x-x^{\prime}-m\right| \leq\left(y y^{\prime}\right)^{1 / 2}}|m|^{2 p-1}|m|^{-4} . \\
& +\left(y y^{\prime}\right)^{2} \sum_{\left|x-x^{\prime}-m\right|>\left(y y^{\prime}\right)^{1 / 2}} \mid
\end{aligned}
$$

The second and third terms on the right are each

$$
\leq\left(y y^{\prime}\right)^{1 / 2} \ll y^{-1 / 8} y^{19 / 8} .
$$

If $y^{\prime}<7 y / 8$ or $y^{\prime}>9 y / 8$, Lemma 1 implies that

$$
\begin{aligned}
\sum_{n} k\left(\tau, U^{n} \tau^{\prime}\right) & \ll\left(y y^{\prime}\right)^{2} \int_{-\infty}^{+\infty}\left\{\left(x-x^{\prime}-t\right)^{2}+\left(y-y^{\prime}\right)^{2}\right\}^{-2} d t \\
& \ll\left(y y^{\prime}\right)^{2}\left|y-y^{\prime}\right|^{-3} .
\end{aligned}
$$

As in the proof of Lemma 2, this term satisfies the stated inequality.

Finally we consider the difference $D\left(\tau, \tau^{\prime}\right)$ defined by

$$
\begin{aligned}
D\left(\tau, \tau^{\prime}\right) & \doteq \sum_{n=-\infty}^{+\infty} k\left(\tau, U^{n} \tau^{\prime}\right)-k_{0}\left(\tau, \tau^{\prime}\right) \\
& =\sum_{n} \int_{0}^{1}\left\{k\left(\tau, U^{n} \tau^{\prime}\right)-k\left(\tau, U^{n+t} \tau^{\prime}\right)\right\} d t .
\end{aligned}
$$

The following lemma contains the estimate for $D$ that we require.

LEMMA 4. If $7 y / 8 \leq y^{\prime} \leq 9 y / 8$ and $y \neq y^{\prime}$ then

$$
\begin{aligned}
& D\left(\tau, \tau^{\prime}\right) \ll\left(y y^{\prime}\right)^{1 / 2-p}+\left(y y^{\prime}\right)^{1 / 2-p} \sum_{|n| \leq 3}\left\{\left(y-y^{\prime}\right)^{2}+\left(x-x^{\prime}-n\right)^{2}\right\}^{p-1 / 2} \\
&+\left(y y^{\prime}\right)^{1 / 2-p} \sum_{|n| \leq 3} \int_{0}^{1}\left\{\left(y-y^{\prime}\right)^{2}+\left(x-x^{\prime}-n-t\right)^{2}\right\}^{p-1 / 2} d t .
\end{aligned}
$$

If $y^{\prime}<7 y / 8$ or $y^{\prime}>9 y / 8$ then $D\left(\tau, \tau^{\prime}\right) \ll 1$.

ProOF. Assume $7 y / 8 \leq y \leq 9 y / 8$ and $y \neq y^{\prime}$. If $|n| \geq 3$ then

$$
\left|k\left(\tau, U^{n} \tau^{\prime}\right)-k\left(\tau, U^{n+t} \tau^{\prime}\right)\right| \ll k^{\prime}(\xi)\left(y y^{\prime}\right)^{-1}\left|x-x^{\prime}-n\right|,
$$


where $\xi=\left(y y^{\prime}\right)^{-1}\left\{\left(y-y^{\prime}\right)^{2}+\left(x-x^{\prime}-n-\eta\right)^{2}\right\}$ with $0 \leq \eta \leq 1$. By Lemma 1 the term on the right is

$$
\ll k_{1}^{\prime}(\xi)\left(y y^{\prime}\right)^{-1}\left|x-x^{\prime}-n\right| .
$$

If $3 \leq|n| \leq\left(y y^{\prime}\right)^{1 / 2}$ then this bound is $\ll\left(y y^{\prime}\right)^{1 / 2-p}|n|^{2 p-2}$. If $|n|>\left(y y^{\prime}\right)^{1 / 2}$, then it is $\ll\left(y y^{\prime}\right)|n|^{-5}$. The terms with $|n| \leq 3$ are estimated directly with Lemma 1 . Since $p<1 / 2$, summing over $n$ gives the stated inequality.

If $y^{\prime}<7 y / 8$ or $y^{\prime}>9 y / 8$ then Lemma 1 implies that

$$
k^{\prime}(\xi)\left(y y^{\prime}\right)^{-1}\left|x-x^{\prime}-n\right| \ll|n|\left\{\left(y-y^{\prime}\right)^{2}+n^{2}\right\}^{-3}\left(y y^{\prime}\right)^{2}
$$

for $|n| \geq 3$. The estimate for $D$ in this case is

$$
\begin{aligned}
D & \ll\left(y y^{\prime}\right)^{2}\left(y-y^{\prime}\right)^{-4}+\left(y y^{\prime}\right)^{2} \sum_{|n| \geq 3}|n|\left\{\left(y-y^{\prime}\right)^{2}+n^{2}\right\}^{-3} \\
& \ll\left(y y^{\prime}\right)^{2}\left(y-y^{\prime}\right)^{-4} \ll 1 .
\end{aligned}
$$

This completes the proofs of the required lemmas for Theorem 1 .

5. Estimation of cusp forms. Define the kernel

$$
K^{*}\left(\tau, \tau^{\prime}\right)=K\left(\tau, \tau^{\prime}\right)-K_{\kappa}\left(\tau, \tau^{\prime}\right),
$$

where $K$ and $K_{i}$ are defined in (4.1) and $\kappa=\kappa(\tau)$ as given in (1.4). We have

$$
K_{\kappa}\left(\tau, \tau^{\prime}\right)=\sum_{\Gamma_{\kappa} \backslash \Gamma} k_{0}\left(\tau_{\kappa}, \tau_{\kappa, M}^{\prime}\right),
$$

where by Lemma 2 each term has the majorization

$$
k_{0}\left(\tau_{\kappa}, \tau_{\kappa, M}^{\prime}\right) \ll y_{\kappa, M}^{\prime 9 / 8}
$$

This estimate allows

$$
\begin{aligned}
\int_{\mathcal{F}} K_{\kappa}\left(\tau, \tau^{\prime}\right) e_{j}\left(\tau^{\prime}\right)\left(d \tau^{\prime}\right) & =\sum_{\Gamma_{i} \backslash \Gamma} \int_{\mathcal{F}} k_{0}\left(\tau_{\kappa}, \tau_{\kappa, M}^{\prime}\right) e_{j}\left(\tau^{\prime}\right)\left(d \tau^{\prime}\right) \\
& =\int_{0}^{\infty} y_{\kappa}^{\prime} d y_{\kappa}^{\prime} \int_{0}^{1} k_{0}\left(\tau_{\kappa}, \tau_{\kappa}^{\prime}\right) e_{j}\left(\tau^{\prime}\right) d x_{\kappa}^{\prime} .
\end{aligned}
$$

Since $k_{0}\left(\tau_{\kappa}, \tau_{\kappa}^{\prime}\right)$ does not depend on $x_{\kappa}^{\prime}$ and the constant term of the expansion of the $e_{j}$ at the $\kappa$ th cusp is zero, it follows that the integral above is zero. Therefore

$$
h\left(r_{j}\right) e_{j}(\tau)=\int_{\mathcal{F}} K^{*}\left(\tau, \tau^{\prime}\right) e_{j}\left(\tau^{\prime}\right)\left(d \tau^{\prime}\right),
$$

hence

$$
\left|e_{j}(\tau)\right|^{2} \leq\left|h\left(r_{j}\right)\right|^{2} \int_{\mathcal{F}}\left|K^{*}\left(\tau, \tau^{\prime}\right)\right|^{2}\left(d \tau^{\prime}\right) .
$$

We will estimate the integral above. For $\tau$ given, and $\kappa=\kappa(\tau)$, set $\mathcal{F}=\mathcal{F}_{1} \cup \mathcal{F}_{2}$, with

$$
\digamma_{1} \doteq\left\{\tau^{\prime} \in \mathcal{F} \mid y_{\kappa}^{\prime} \geq C_{0} / 2\right\}, \quad \digamma_{2} \doteq\left\{\tau^{\prime} \in \mathcal{F} \mid y_{\kappa}^{\prime}<C_{0} / 2\right\}
$$

Also let

$$
S_{\tau}=\left\{\tau^{\prime} \in \mathcal{F} \mid 7 y_{\kappa} / 8 \leq y_{\kappa}^{\prime} \leq 9 y_{\kappa} / 8\right\}
$$


If $\tau^{\prime} \in F_{2}$, then from Lemma 3

$$
K\left(\tau, \tau^{\prime}\right)=\sum_{\substack{M \\ \Gamma_{\kappa} \backslash \Gamma}}\left(\sum_{n} k\left(\tau_{\kappa}, U^{n} \tau_{\kappa, M}^{\prime}\right)\right) \ll \sum_{\Gamma_{\kappa} \backslash \Gamma} y_{\kappa, M}^{\prime 9 / 8} \ll 1
$$

The same estimate for $K_{\kappa}$ follows from (5.2), thus

$$
K^{*}\left(\tau, \tau^{\prime}\right) \ll 1 \quad \text { for } \tau^{\prime} \in \mathcal{F}_{2} .
$$

If $\tau^{\prime} \in \mathcal{F}_{1}$, write

$$
\begin{aligned}
\left|K^{*}\left(\tau, \tau^{\prime}\right)\right|^{2} & \ll\left|D\left(\tau_{\kappa}, \tau_{\kappa}^{\prime}\right)\right|^{2}+\left|\sum_{M \neq \mathrm{id}} \sum_{n} k\left(\tau_{\kappa}, u^{n} \tau_{\kappa, M}^{\prime}\right)\right|^{2}+\left|\sum_{M \neq \mathrm{id}} k_{\kappa}\left(\tau, \tau^{\prime}\right)\right|^{2} \\
& \doteq|D|^{2}+\left|\sum_{1}\right|^{2}+\left|\sum_{2}\right|^{2} .
\end{aligned}
$$

Lemma 2 and (2.4) show

$$
\sum_{2} \ll \sum_{M \neq \mathrm{id}} y_{\kappa, M}^{9 / 8} \ll 1
$$

for $\tau^{\prime} \in \mathcal{F}$. Lemma 3 and (2.4) give

$$
\sum_{1}^{5.6} \ll 1+y_{\kappa}^{1-2 p} \sum_{\substack{7 y_{\kappa} / 8 \leq y_{\kappa, M} \leq 9 y_{\kappa} / 8 \\ M \neq \text { id }}} \sum_{|n| \leq 3}\left\{\left(x_{\kappa}-x_{\kappa, M}^{\prime}-n\right)^{2}+\left(y_{\kappa}-y_{\kappa, M}^{\prime}\right)^{2}\right\}^{p-1 / 2}
$$

Note that the first sum on the right of (5.6) can contain at most finitely many terms since $y_{\kappa} \geq C_{0}>0$. The number of terms is bounded above by a constant that is independent of $y_{\kappa}^{\prime}$. If $y_{\kappa} \geq C_{1}$, for some sufficiently large $C_{1}>1$ then the sum contains no terms. It follows that

$$
\int_{\mathcal{F}}\left|\sum_{1}\right|^{2}\left(d \tau^{\prime}\right) \ll 1+C_{1}^{2} \sum \sum \int_{A_{\kappa} M \mathcal{F}_{1}}\left\{\left(x_{\kappa}-x_{\kappa}^{\prime}-n\right)^{2}+\left(y_{\kappa}-y_{\kappa}^{\prime}\right)^{2}\right\}^{2 p-1}\left(d \tau_{\kappa}^{\prime}\right) .
$$

The integrals on the right are finite if $p>0$. It follows that

$$
\int_{\mathcal{F}_{1}}\left|K^{*}\right|^{2}\left(d \tau^{\prime}\right) \ll 1+\int_{\mathcal{F}_{1}}|D|^{2}\left(d \tau^{\prime}\right) .
$$

Lemma 4 shows

$$
\int_{\mathcal{F}_{\mathfrak{1}} \backslash S_{\tau}}|D|^{2}\left(d \tau^{\prime}\right) \ll 1
$$


and

$$
\begin{aligned}
& \int_{S_{\tau}}\left|D\left(\tau, \tau^{\prime}\right)\right|^{2}\left(d \tau^{\prime}\right) \ll y_{\kappa}^{-4 p} \int_{0}^{1} d x_{\kappa}^{\prime} \int_{7 y_{\kappa} / 8}^{9 y_{\kappa} / 8} d y_{\kappa}^{\prime} \\
& +y_{\kappa}^{-4 p} \sum_{|n| \leq 3} \int_{0}^{1} d x_{\kappa}^{\prime} \int_{7 y_{\kappa} / 8}^{9 y_{\kappa} / 8}\left\{\left(y_{\kappa}-y_{\kappa}^{\prime}\right)^{2}+\left(x_{\kappa}-x_{\kappa}^{\prime}-n\right)^{2}\right\}^{2 p-1} d y_{\kappa}^{\prime} \\
& +y_{\kappa}^{-4 p} \sum_{|n| \leq 3} \int_{0}^{1} d t \int_{0}^{1} d x_{\kappa}^{\prime} \int_{7 y_{\kappa} / 8}^{9 y_{\kappa} / 8}\left\{\left(y_{\kappa}-y_{\kappa}^{\prime}\right)^{2}+\left(x_{\kappa}-x_{\kappa}^{\prime}-n-t\right)^{2}\right\}^{2 p-1} d y_{\kappa}^{\prime} \\
& <C(p) y_{\kappa}^{1-4 p} .
\end{aligned}
$$

It follows from (5.2), (5.4), (5.5) and (5.7)-(5.9) that

$$
\left|e_{j}(\tau)\right|^{2}<C(p)\left(1+r_{j}^{2}\right)^{2 p+1}\left(1+y_{\kappa}^{1-4 p}\right) .
$$

Setting $\alpha=p+1 / 2$ and again noting that $y_{\kappa} \geq C_{0}$ gives Theorem 1 as stated.

6. Remarks. It might be possible to improve the estimate of Theorem 1 by choosing $h(r)$ to be large locally near the desired value of $r_{j}$. I have not been able to do as well as Theorem 1 with this approach.

It is known, via the methods in [1], that it is not possible to replace the factor $e^{\pi r_{j} / 2}$ in Theorem 3 by $e^{a r_{j}}$ with $a<\pi / 2$.

\section{REFERENCES}

1. R. W. Bruggeman, Fourier coefficients of cusp forms, Invent. Math. 45 (1978), 1-18.

2. T. Kubota, Elementary theory of Eisenstein series, Halstead Press, New York, 1973.

3. H. Maass, Über eine neue Art von nichtanalytischen automorphen Funktionen und die Bestimmung Dirichletscher Reihen durch Funktionalgleichungen, Math. Ann. 121 (1949), 141-183.

4. H. Neunhoffer, Über die Analytische Fortsetzung von Poincareneihen, S-B Heidelberger Akad. Wiss. Math-Nat. Kl. Abh. 2 (1973).

5. W. Roelcke, Des Eigenwertproblem der automorphen Formen in der hyperbolischen Ebene. II, Math. Ann. 168 (1967), 261-324.

6. A. Selberg, Harmonic analysis and discontinuous groups in weakty symmetric Riemannian spaces with applications to Dirichlet series, J. Indian Math. Soc. 20 (1956), 47-87.

Department of Mathematics, Lehigh University, Bethlehem, Pennsylvania 18015

Current address: Box 265, Graysville, Alabama 35073 